\title{
Silver nanoislands on cellulose fibers for chromatographic separation and ultrasensitive detection of small molecules
}

\author{
Hyukjin Jung, Moonseong Park, Minhee Kang and Ki-Hun Jeong
}

High-throughput small-molecule assays play essential roles in biomedical diagnosis, drug discovery, environmental analysis, and physiological function research. Nanoplasmonics holds a great potential for the label-free detection of small molecules at extremely low concentrations. Here, we report the development of nanoplasmonic paper (NP-paper) for the rapid separation and ultrasensitive detection of mixed small molecules. NP-paper employs nanogap-rich silver nanoislands on cellulose fibers, which were simply fabricated at the wafer level by using low-temperature solid-state dewetting of a thin silver film. The nanoplasmonic detection allows for the scalable quantification and identification of small molecules over broad concentration ranges. Moreover, the combination of chromatographic separation and nanoplasmonic detection allows both the highly sensitive fluorescence detection of mixed small molecules at the attogram level and the label-free detection at the sub-nanogram level based on surface-enhanced Raman scattering. This novel material provides a new diagnostic platform for the high-throughput, low-cost, and label-free screening of mixed small molecules as an alternative to conventional paper chromatography.

Light: Science \& Applications (2016) 5, e16009; doi:10.1038/lsa.2016.9; published online 15 January 2016

Keywords: nanoplasmonics; paper chromatography; plasmon-enhanced spectroscopy; silver nanoislands; small-molecule assay

\section{INTRODUCTION}

Small molecules ( $<900 \mathrm{Da})$ include cell-signaling molecules, metabolites, pharmaceuticals, and many other organic compounds. They play important roles both in the diverse biochemical functions of a living organism and in the development of therapeutic agents ${ }^{1-3}$. For instance, vitamins are crucial to cell regulation, tissue growth and differentiation, metabolic activities, and DNA repair as a pharmaceutical function ${ }^{4}$. Consequently, the detection and quantification of small molecules are crucial for biomedical diagnosis, drug discovery, environmental analysis, and physiological function research ${ }^{1-7}$. For decades, noncompetitive immunoassays ${ }^{5}$, high-performance liquid chromatography ${ }^{6}$, and capillary electrophoresis ${ }^{7}$ have been extensively developed for separating low-molecular-weight analytes. However, their experimental complexities and bulky instrumentation hinder rapid, simple, and affordable small-molecule assays; therefore, interest has increased in developing reliable alternatives that can be used in high-throughput screening techniques.

Paper, which is one of the most widespread organic biomaterials found in nature, is a simple and cost-effective platform for smallmolecule assays. The abundant porosity and surface charges on the cellulose fiber matrices allow for the selective adsorption of analyte molecules from a small volume solution using capillary action (e.g., without external pumping) to yield attractive extraction or separation ${ }^{8,9}$. For example, paper chromatography and dot-immunobinding assays often utilize pure cellulose or nitrocellulose paper as a support for biochemical screening ${ }^{9-11}$. More recently, paper-based biosensors have progressively contributed to the sensitive detection of small molecules such as glucose ${ }^{12}$, neurotoxins ${ }^{13}$, and $\mathrm{DNA}^{14}$. Such biosensors utilize either the specific binding of target molecules to enzymes or enzyme-conjugated binding of antibodies to antigens immobilized on the cellulose fiber matrices and thereafter collect a colorimetric readout using labeled reagents such as redoxactive ions or fluorophores ${ }^{12-14}$. However, paper-based biosensors are still under development for use in the high-throughput screening of small molecules at low concentrations.

Nanoplasmonics have recently spurred the highly sensitive detection of small molecules at ultralow concentrations. The collective oscillation of free electrons near noble metal nanostructures - 'localized surface plasmon resonance (LSPR)' - significantly enhances light-matter interactions, with an intense electromagnetic (EM) field termed a 'hot spot' ${ }^{15-17}$. This new approach extensively contributes to the highly sensitive spectroscopic techniques of biochemical molecules such as surface-enhanced Raman scattering (SERS) or metal-enhanced fluorescence (MEF) at the single molecule level ${ }^{18,19}$. However, the nanoplasmonic sensing of small molecules at low concentrations is challenging because of their low adsorption onto metallic nanostructures. Furthermore, microfluidic motion (i.e., thermodiffusion and convection) upon the incidence of light may hinder 
molecular accumulation near the hot $\operatorname{spots}^{20,21}$. Consequently, molecular confinement near multiple hot spots is apparently a prerequisite for ultrasensitive plasmonic detection ${ }^{22-24}$. As a new biosensing substrate, the cellulose fiber matrices of paper can effectively resolve this technical challenge owing to their structural hierarchy, extraordinary hygroscopy, and high physicochemical adsorption. Over the last decade, metal nanostructures on cellulose fiber matrices have been employed by incorporating chemical synthesis, ink jetting, laser annealing, and direct writing ${ }^{25-28}$. However, all of the previous methods involve either complicated aqueous processes or the local implementation of metal nanostructures on the cellulose fiber matrices; thus, they still have some technical limitations for the practical use of chromatographic separation and the ultrasensitive detection of small molecules.

Here, we report the use of nanoplasmonic paper (NP-paper) for the chromatographic separation of mixed small molecules and their ultrasensitive detection. NP-paper employs nanogap-rich silver nanoislands on the top surface of cellulose fiber matrices, which can serve not only as a chromatographic column for the separation of small molecules in aqueous solution but also as a sensing substrate for highly sensitive nanoplasmonic detection. The differential partitioning of small molecules can be driven rapidly under capillary action, depending on the retention factor, i.e., the solubility and the polarity difference related to the solvent. The separated molecules also reside near the nanogap-rich silver nanoislands, which allow the plasmonic enhancement of optical signatures such as fluorescence or SERS signals.

\section{MATERIALS AND METHODS}

\section{Chemicals}

Crystal violet (CV), toluidine blue (TB), Congo red (CR), safranin O (SO), fluorescein isothiocyanate (FITC), (-)-riboflavin, $\beta$-carotene, $( \pm)$ - $\alpha$-tocopherol, pyridine $(99.8 \%)$, and $n$-hexane $(99 \%)$ were obtained from Sigma-Aldrich Korea Ltd. (Yongin-si, Gyeonggi-do, Korea) and used without further purification. Ethanol (99.5\%), methanol (99.9\%), and isopropyl alcohol (99.5\%) were obtained from Junsei Chemical Co. Ltd. (Tokyo, Japan).

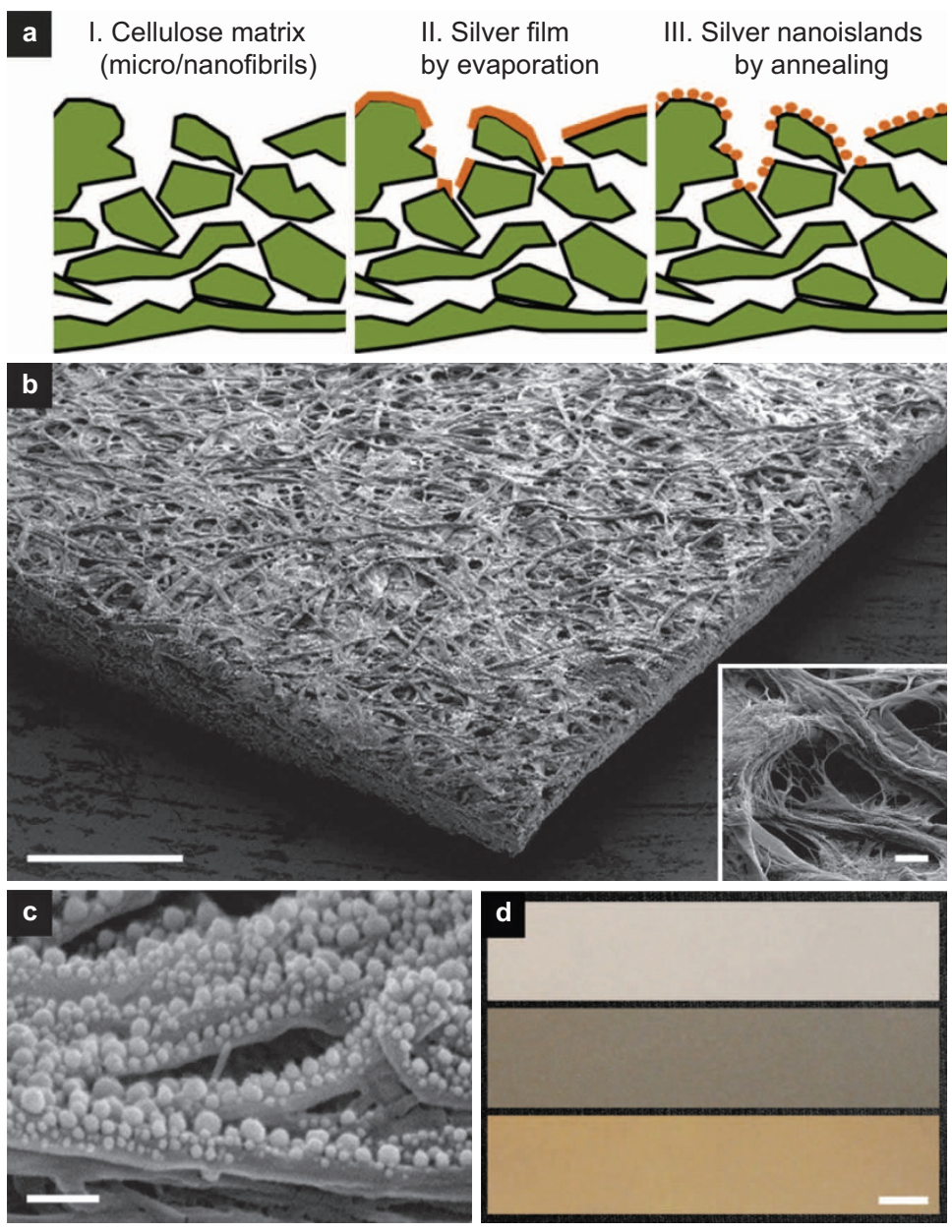

Figure 1 Nanoplasmonic paper (NP-paper). (a) Large-area and batch nanofabrication of nanogap-rich silver nanoislands on cellulose fiber matrices using solid-state dewetting of a thin silver film. The thermally evaporated thin silver film on the cellulose fiber forms nanogap-rich silver nanoislands after thermal annealing at low temperatures. (b) A perspective-view scanning electron microscope (SEM) image of NP-paper (scale bar: $500 \mu \mathrm{m}$ ). The cellulose fibers possess a complex structural hierarchy from nanofibrils (20-200 nm in diameter) to microfibrils (5-20 $\mu \mathrm{m}$ in diameter). The inset shows a magnified SEM image of cellulose nano/microfibrils with abundant micropores (scale bar: $10 \mu \mathrm{m}$ ). (c) A cross-sectional SEM image of NP-paper. The top surface is only covered with nanogap-rich silver nanoislands, which maintain their hygroscopic nature and the physicochemical adsorption of the cellulose fiber matrices but strongly enhance light confinement on the surface (scale bar: 200 nm). (d) Photographic images of tailored normal chromatography paper (top), paper with thin silver film (middle), and 'NP-paper' with silver nanoislands (bottom) (scale bar: $10 \mathrm{~mm}$ ). 


\section{Nanoisland implementation on paper at the wafer level}

Large-scale NP-paper was fabricated by using low-temperature solidstate dewetting of a thin silver film on paper (Figure 1). A 0.18-mmthick, Whatman cellulose chromatography paper (Sigma-Aldrich) was prepared as a substrate. First, a thin silver film grown in the VolmerWeber mode ${ }^{29}$ was thermally evaporated on a 4 -inch paper (chamber pressure: $3 \sim 6 \mu$ Torr, deposition rate: $0.1 \mathrm{~nm} \mathrm{~s}^{-1}$ ). The thin film of silver was then transformed into nanoislands by thermally annealing on a hot plate at $160{ }^{\circ} \mathrm{C}$ for 30 min under ambient conditions.

\section{Numerical analysis of electric field distribution}

The electric field from the silver nanoislands on cellulose fibers was numerically calculated by using a three-dimensional finite-difference time-domain method (Lumerical FDTD Solutions 8.0.6, Lumerical Solutions, Inc., Vancouver, Canada), depending on the initial thickness of the thin silver film (Figure 2a). The binary segments of the silver nanoislands were extracted from scanning electron microscope (SEM) images using the Image $\mathrm{J}$ software (National Institute of Health, Bethesda, MD, USA), and each silver nanoisland was considered a cylindrical shape for the 3D FDTD analysis.

\section{SERS enhancement factor (EF) of NP-paper}

The SERS and Raman signals of the CV molecules were compared between NP-paper and normal chromatography paper. In the experiment, the molar concentration of CV was $50 \mathrm{nM}(0.1 \mu \mathrm{L})$ for SERS and $500 \mu \mathrm{M}(0.1 \mu \mathrm{L})$ for Raman. SERS EF was calculated by the conventional method ${ }^{30}$. SERS EF is $I_{\mathrm{SERS}} \mathcal{c}_{\mathrm{RS}} H_{\mathrm{eff}} / I_{\mathrm{RS}} \mu_{\mathrm{M}} \mu_{\mathrm{S}} A_{\mathrm{M}}$, where $I_{\mathrm{SERS}}$ is the SERS intensity, $c_{\mathrm{RS}}$ is the molar concentration in the Raman experiment, $H_{\text {eff }}$ is the effective height of scattering, $I_{\mathrm{RS}}$ is the Raman signal intensity, $\mu_{\mathrm{M}}$ is the surface density of the individual metal nanostructure, $\mu_{\mathrm{S}}$ is the surface density of the $\mathrm{CV}$ adsorbents on the metal, and $A_{\mathrm{M}}$ is the metallic surface area in each surface. $I_{\mathrm{SERS}}$ and $I_{\mathrm{RS}}$ were obtained at $1590 \mathrm{~cm}^{-1}$ under the laser excitation (wavelength: $488 \mathrm{~nm}$, power: $1.5 \mathrm{~mW}$, and integration time: $1 \mathrm{~s}$ ). $H_{\text {eff }}$ was also experimentally measured in the Raman signal measurement. $\mu_{\mathrm{M}}$ and $A_{\mathrm{M}}$ were analyzed from an SEM image under the assumption that each silver nanoisland has a hemispherical shape. $\mu_{\mathrm{S}}$ was calculated under the assumption that the CV molecules are uniformly distributed over the NP-paper. The SERS EF is $1.91 \times 10^{7}$.

\section{Small-molecule detection on NP-paper}

The SERS signals, LSPR wavelength shift, and extinction spectra of CV molecules were measured from a single spot on NP-paper fabricated from an initial thickness of $10 \mathrm{~nm}$ using a spectrometer (MicroSpec2300i, Princeton Instrument, Trenton, NJ, USA) with an inverted microscope (Axiovert 200M, Carl Zeiss Co. Ltd. Seoul, Korea). A volume of $0.1 \mu \mathrm{L} \mathrm{CV}$ solution in water was micropipetted on $\mathrm{NP}$-paper and evaporated under ambient conditions for $10 \mathrm{~min}$ (Figure 3a). The concentration of CV solution ranged from $10 \mathrm{nM}$ to $1 \mathrm{mM}$, which corresponded to a molecular quantity from $408 \mathrm{fg}$ to 40.8 ng per unit spot. The SERS signals were measured with an argon ion laser (wavelength: $488 \mathrm{~nm}$, power: $0.5 \mathrm{~mW}$ ) coupled to a $50 \times$ objective lens (numerical aperture: 0.5 ). The integration time was $0.5 \mathrm{~s}$ in a spectrometer. In the LSPR and extinction experiments, the transmission $T$ was measured from NP-paper and normalized with that from a normal chromatography paper. The extinction values were then calculated as $1-T_{\mathrm{NP}-\text { paper }+\mathrm{CV}} / T_{\text {paper }}$, and the extinction of the CV adsorbed on normal paper was calculated as $1-T_{\text {paper }+\mathrm{CV}} /$ $T_{\text {paper. }}$ In the MEF experiment, stock solutions of FITC, SO, CR, and TB were prepared in deionized water. First, $0.1 \mu \mathrm{L}$ of the analytical solutions was spotted on NP-paper and then dried under vacuum conditions for $10 \mathrm{~min}$. The molar concentration of the individual sample solution was $500 \mathrm{nM}$, which corresponds to the molecular quantity of $50 \mathrm{fmol}$ per unit spot. The fluorescence spectra of each molecule on NP-paper were characterized with an inverted confocal laser scanning microscope by sequentially imaging the optical sections. The lateral optical section of $900 \mu \mathrm{m} \times 900 \mu \mathrm{m}$ was stacked along the light propagation axis (z-axis) over a range of $20 \mu \mathrm{m}$, with an interval of $1 \mu \mathrm{m}$. For the fluorescence emission of each dye molecule, the laser power (excitation wavelength, $\lambda_{\text {ex }}: 488 \mathrm{~nm}, 514 \mathrm{~nm}$, and $633 \mathrm{~nm}$ ) in the focus of the microscope objective lens (10×, numerical aperture: 0.3 ) was kept constant at $60 \mu \mathrm{W}$. Fluorescence images were
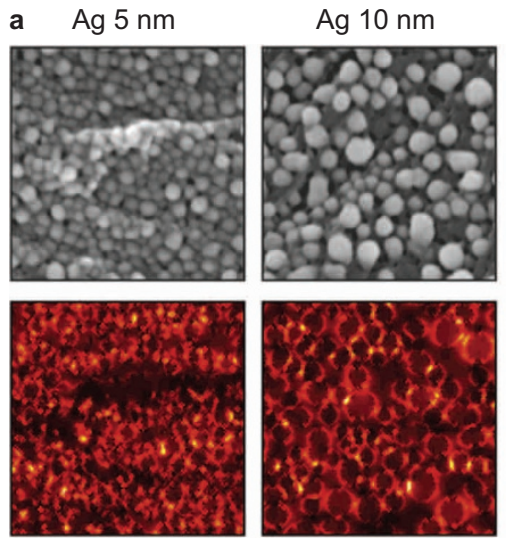

$\operatorname{Ag} 15 \mathrm{~nm}$

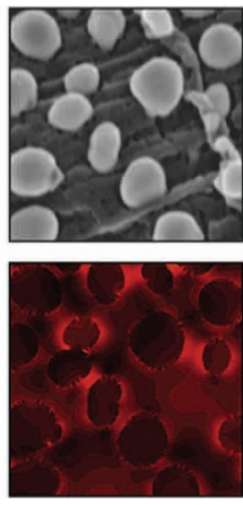

$\operatorname{Ag} 20 \mathrm{~nm}$
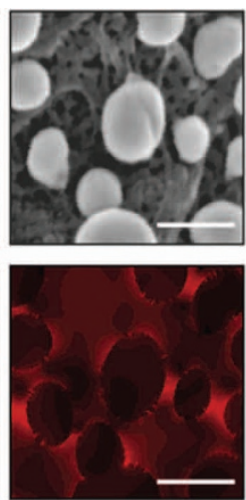

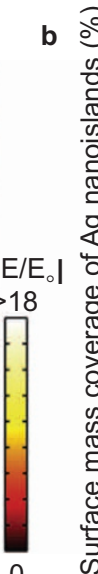

0

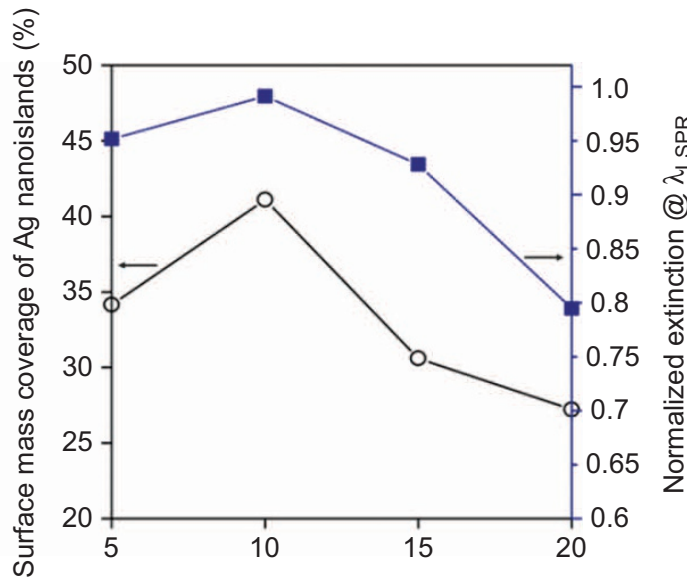

Initial silver film thickness $(\mathrm{nm})$

Figure 2 Multiple EM hot spots from nanogap-rich silver nanoislands on cellulose fiber matrices. (a) Top-view SEM images (top) and electric field distributions derived from geometrical configurations of SEM images (bottom) of size-controlled silver nanoislands on cellulose fiber matrices (scale bar: $200 \mathrm{~nm}$ ). The average diameter and the interstitial gap spacing of the silver nanoislands moderately increase with the initial silver film thickness $(5 \mathrm{~nm}, 10 \mathrm{~nm}, 15 \mathrm{~nm}$, and $20 \mathrm{~nm})$ before thermal annealing. Based on the calculated FDTD results, the electric field at the LSPR wavelength $\left(\lambda_{\text {LSPR }}\right)$ is locally enhanced by a factor of 24 for highly dense and nanogaprich silver nanoislands formed from the initial Ag thickness of $10 \mathrm{~nm}$. (b) The relationship between the surface mass coverages of the silver nanoislands and their extinction intensities measured at the individual plasmon resonance wavelengths. The maximum surface mass coverage and extinction at the LSPR wavelength are obtained from the initial silver thickness of $10 \mathrm{~nm}$. 
acquired through spectral filters of $505-530 \mathrm{~nm}$ for FITC $\left(\lambda_{\text {ex }}: 488\right.$ $\mathrm{nm}), 530-600 \mathrm{~nm}$ for SO ( $\left.\lambda_{\text {ex }}: 488 \mathrm{~nm}\right), 560-615 \mathrm{~nm}$ for CR $\left(\lambda_{\text {ex }}: 514\right.$ $\mathrm{nm})$, and $>650 \mathrm{~nm}$ for TB $\left(\lambda_{\text {ex }}: 633 \mathrm{~nm}\right)$. The fluorescence EFs were then determined by dividing the average pixel intensities of the analytes adsorbed on the NP-paper by those on normal paper obtained from the image histograms. The limit of detection (LOD) is the smallest concentration or absolute amount of analyte that has a signal significantly larger than the signal arising from an analyte blank.
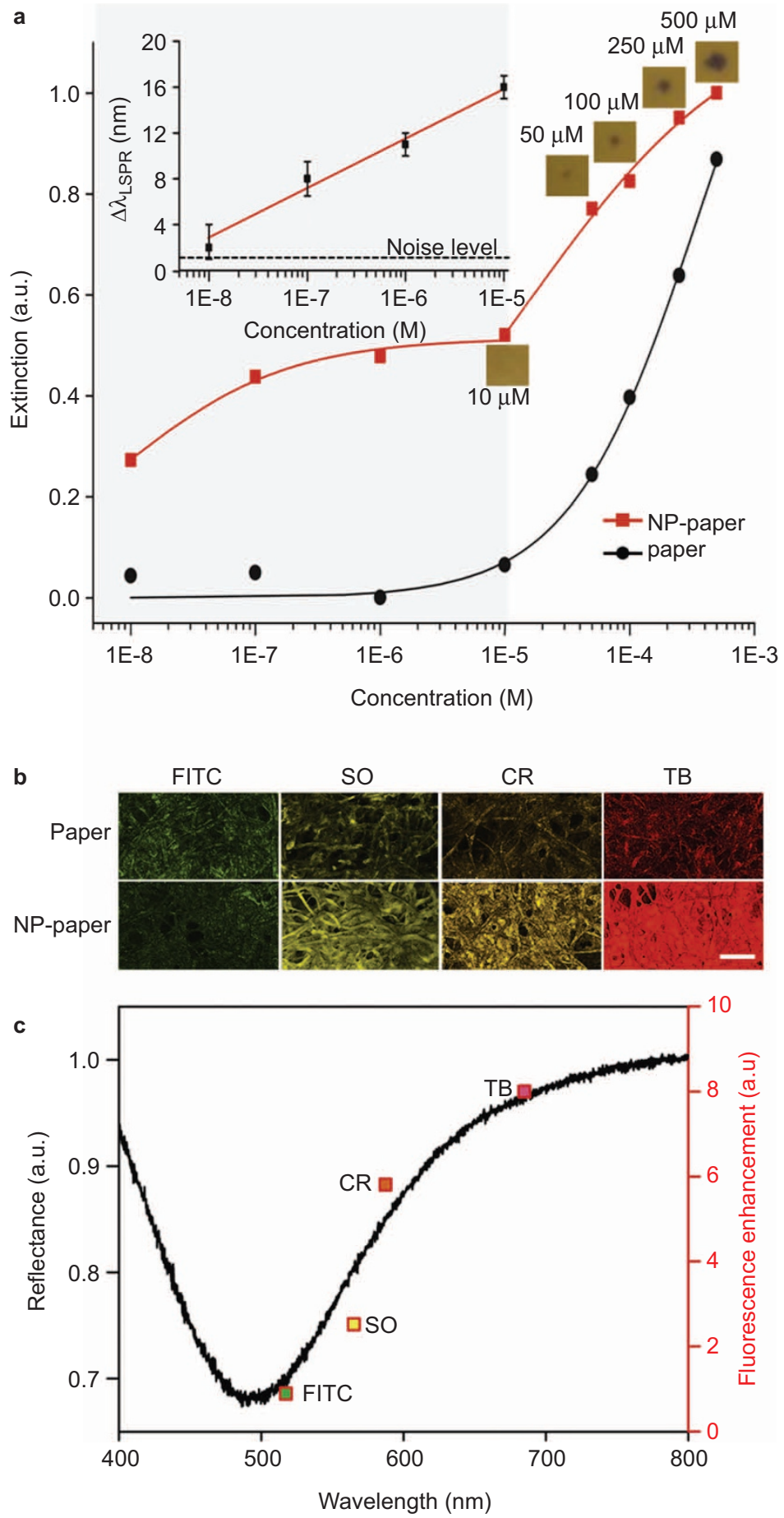

Figure 3 Ultrasensitive detection of small molecules. (a) Extinction at the absorption band (wavelength: $600 \mathrm{~nm}$ ) of CV spotted on chromatography paper with (red solid rectangle) and without (black solid circle) silver nanoislands, depending on the solution concentration. For NP-paper, both the LSPR shift and the extinction quantitatively indicate the concentration of small molecules. CV molecules were detected from the extinction spectra for high concentrations ranging from $10 \mu \mathrm{M}$ to $500 \mu \mathrm{M}$ (optical micrographs: CV adsorbed on NP-paper) and even from the LSPR wavelength shift at lower concentrations from $50 \mathrm{nM}$ to $10 \mu \mathrm{M}$ (inset: $\lambda_{\mathrm{LSPR}}$ shift vs. the concentration), whereas normal paper can detect the extinction at over $10 \mu \mathrm{M}$ in concentration. (b) Comparison of fluorescence emissions captured from FITC, SO, $\mathrm{CR}$, and TB dye molecules adsorbed on paper without (top) and with (bottom) silver nanoislands (scale bar: $200 \mu \mathrm{m}$ ). (c) Fluorescence enhancement of FITC, SO, CR, and TB molecules at $500 \mathrm{nM}$. The fluorescence intensities are increased by a factor of 8 for TB, and the enhancement ratios for the individual molecules strongly correlate with the reflectance spectra of the silver nanoislands measured from NP-paper. 
The LOD of NP-paper was calculated by the IUPAC standard as

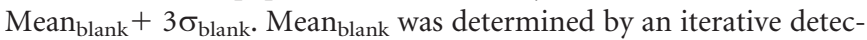
tion with 18 cycles using an NP-paper background only.

\section{Separation and plasmonic detection with NP-paper}

Stock solutions of CV, SO, TB, and CR were prepared in deionized water. The mixed solution with a volume of $0.1 \mu \mathrm{L}$ was spotted on NPpaper and then dried under ambient conditions. The molecules adsorbed on NP-paper were $1 \mu \mathrm{g}$ for CR $(50 \mathrm{mM}), 499 \mathrm{ng}$ for TB (40 $\mathrm{mM})$, and $68 \mathrm{ng}$ for $\mathrm{CV}(5 \mathrm{mM})$ in molecular quantity (Figure 4a). All ascending chromatograms were developed in an air- tight bottle by using 99.5\% ethanol (Figure 4a) and 95\% ethanol (Figure 4b) as a mobile phase. Each experiment for chromatography took $15 \mathrm{~min}$. The NP-paper was then dried under vacuum conditions for $10 \mathrm{~min}$. All of the SERS spectra from the dye molecules were acquired by subtracting the intrinsic SERS spectrum of the NP-paper. These antioxidant vitamins are light-sensitive and easily oxidized when exposed to an oxygen-rich environment, so the sample preparation and separation were carefully performed under subdued light. Stock solutions were prepared for (-)-riboflavin (380 $\mathrm{mg} \mathrm{L}^{-1}$; $1 \mathrm{mM})$ in $80 \%$ pyridine, for $\beta$-carotene $\left(27 \mathrm{mg} \mathrm{L}^{-1} ; 50 \mu \mathrm{M}\right)$ in $\mathrm{n}$-hexane, and for $( \pm)$ - $\alpha$-tocopherol $\left(900 \mathrm{mg} \mathrm{L}^{-1} ; 2 \mathrm{mM}\right)$ in ethanol.
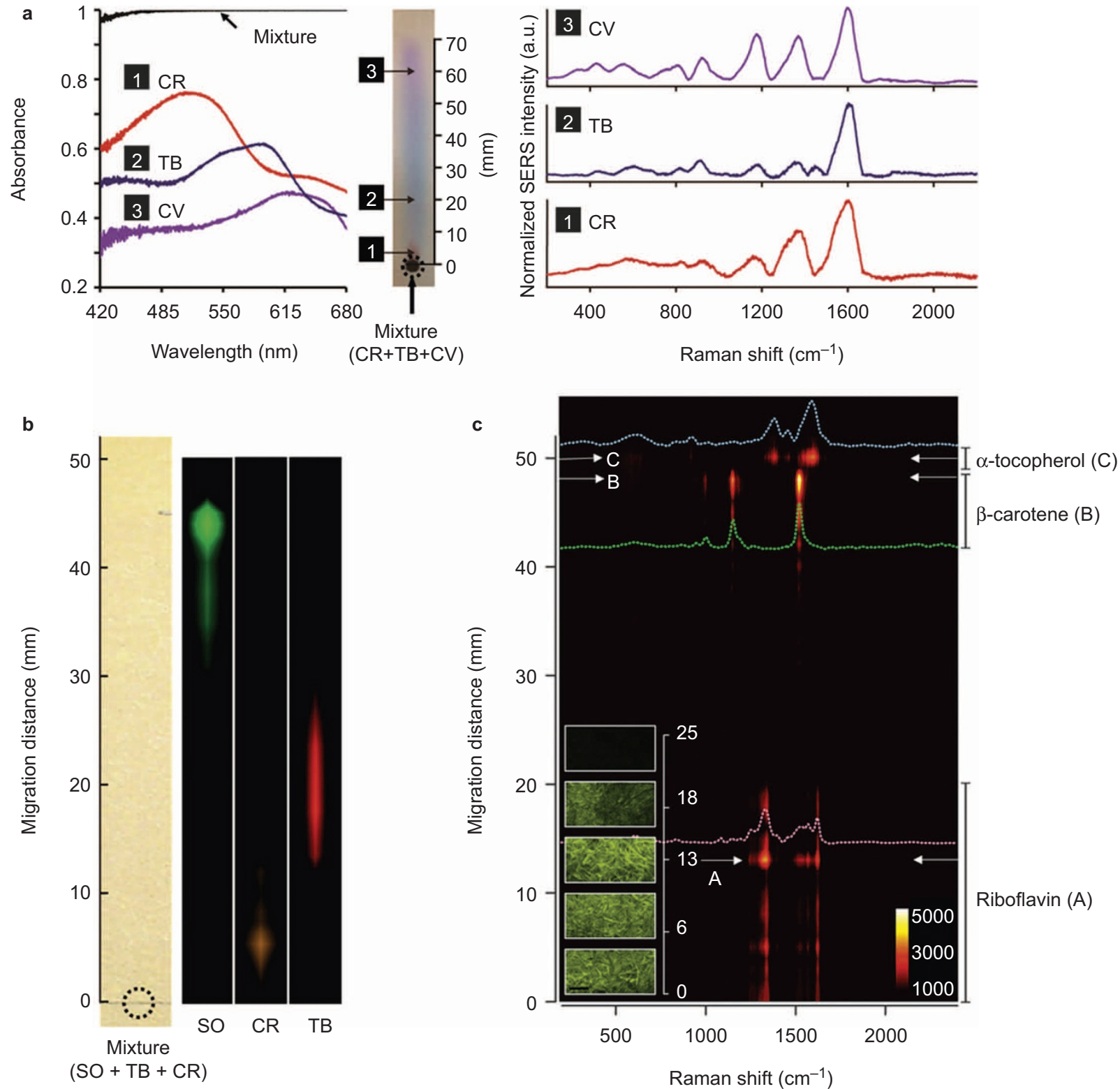

Figure 4 Colorimetric, MEF, and SERS detections after small-molecule separation. (a) Colorimetric detection of dye molecules (CR, TB, and CV) in an aqueous mixture after chromatographic separation on NP-paper. The separated molecules of CR, TB, and CV at the 1,2, and 3 positions, respectively, are clearly distinguished and also confirmed by the corresponding absorption peaks. The SERS measurement also enables label-free detection after chromatographic separation. (b) Fluorescence detection of mixed dye molecules at ultra-low concentration after the chromatographic separation. The fluorescence signals scanned along the column successfully demonstrate the clear partitioning of SO, TB, and CR dye molecules at sub-picogram levels adsorbed on NP-paper. The retention factors of CR, TB, and SO are 0.13, 0.48 , and 0.96 , respectively. (c) Chromatographic SERS of mixed vitamins (one water-soluble riboflavin of $10 \mathrm{ng}$ and two fat-soluble $\beta$-carotene of 537 pg and $\alpha$ tocopherol of $11 \mathrm{ng}$ ). The SERS spectra of the vitamins are plotted at their maxima on the intensity map. The inset indicates the fluorescence micrographs of riboflavin measured at 530-600 $\mathrm{nm}$ in wavelength (scale bar: $300 \mu \mathrm{m}$ ). 
All stock solutions were stored at $-20{ }^{\circ} \mathrm{C}$. The concentrations of the sample solutions for riboflavin, $\beta$-carotene, and $\alpha$-tocopherol were $250 \mu \mathrm{M}, 10 \mu \mathrm{M}$, and $250 \mu \mathrm{M}$, respectively. Each sample solution with a volume of $0.1 \mu \mathrm{L}$ was spotted on NP-paper and then dried under ambient conditions. All ascending partitions were developed by using a 10:1 (v/v) mixture of methanol and isopropyl alcohol as the mobile phase for $9 \mathrm{~min}$. The NP-paper was then dried under vacuum conditions for $10 \mathrm{~min}$. For the SERS experiments, an argon ion laser (wavelength: $488 \mathrm{~nm}$, power: $0.3 \mathrm{~mW}$ ) was coupled to the $50 \times$ objective lens (numerical aperture: 0.5 ). The integration time was $3 \mathrm{~s}$ in a spectrometer. For the fluorescence experiment of riboflavin, the laser power $\left(\lambda_{\text {ex }}: 488 \mathrm{~nm}\right)$ in the focus of the $10 \times$ objective lens (numerical aperture: 0.3 ) was also kept constant at $60 \mu \mathrm{W}$. Both the MEF and SERS spectra of the vitamins were acquired by subtracting the intrinsic fluorescence and SERS intensities of the NP-paper. The highest SERS intensity of each vitamin was compensated to the identical intensity for the intensity mapping of the SERS spectra (Figure 4c).

\section{RESULTS AND DISCUSSION}

The batch nanofabrication of NP-paper (Figure 1a) was successfully performed by using solid-state dewetting of a thin silver film thermally evaporated on a chromatography paper (see the details in the section on Materials and methods). The ultrastructures of cellulose fiber matrices with silver nanoislands were observed by using a field emission-scanning electron microscope (Sirion, FEI Company, Hillsboro, OR, USA). Figure 1b shows the complex hierarchical structures of the NP-paper and its abundant pores. The cellulose fibers can serve as a column substrate that contains mostly bound microfibrils with diameters in the range of 5-20 $\mu \mathrm{m}$, which in turn comprise nanofibrils (20-200 $\mathrm{nm}$ in diameter). In particular, nanogap-rich silver nanoislands exist on the top surfaces of the nano/microfibrils, which maintain their hygroscopic nature for fluid motion but also strongly localize incident light for ultrasensitive detection (Figure 1c). The photographic images clearly distinguish light scattering from conventional, silver film-coated, and NP-papers (tailored area: $20 \mathrm{~mm} \times 80 \mathrm{~mm}$ ) (Figure 1d and Supplementary Fig. S1).

Nanogap-rich silver nanoislands create multiple EM hot spots on the top surface of the cellulose fiber matrices. The size distribution of the silver nanoislands was moderately controlled by the initial silver film thickness before thermal annealing (Figure 2a). The nanoisland diameter increased from $24 \mathrm{~nm}$ to $175 \mathrm{~nm}$, on average, as the initial silver film increased from $5 \mathrm{~nm}$ to $20 \mathrm{~nm}$ in thickness (see also Supplementary Fig. S2a). The extinction measurements were obtained by comparing the transmission through the cellulose fibers with and without silver nanoislands in the visible spectrum (Supplementary Fig. S2b). The experimental results show that the LSPR wavelength $\left(\lambda_{\text {LSPR }}\right)$ strongly depends on both the diameter and the interstitial gap spacing of the silver nanoislands. Specifically, $\lambda_{\text {LSPR }}$ is red-shifted by $5-10 \mathrm{~nm}$ in the initial silver film thickness due to the increase in the nanoisland diameter and is blue-shifted by $10-20 \mathrm{~nm}$ due to the increase in the interstitial gap ${ }^{31}$. The electric field enhancement was directly calculated from the binary segmentation of the silver nanoislands in the SEM images using an FDTD method (Figure 2a, bottom). NP-paper with an initial film thickness of $10 \mathrm{~nm}$ has a maximum local field enhancement of 24 at a $\lambda_{\text {LSPR }}$ of $482 \mathrm{~nm}$ (Figure 2a, bottom and Supplementary Fig. S2b). The experimental results also show that the extinction at a corresponding $\lambda_{\text {LSPR }}$ has a maximum value when the surface mass coverage of the silver nanoislands becomes maximized (Figure 2b).

NP-paper with multiple EM hot spots allows the nanoplasmonic sensing of small molecules at extremely low concentration.
Plasmon-enhanced optical signatures $\left(\Delta \lambda_{\text {LSPR }}\right.$, SERS, and MEF) were measured from the reference dye molecules. The LSPR wavelength shift $\left(\Delta \lambda_{\text {LSPR }}\right)$ is extremely sensitive to changes in the refractive index of the local dielectric environment, which is highly related to the concentration of solute adsorbed on the plasmonic nanostructures ${ }^{15}$. Figure $3 \mathrm{a}$ shows the extinction values of $\mathrm{CV}$ at $600 \mathrm{~nm}$, measured from papers with and without silver nanoislands, depending on the solution concentration (see the measured extinction spectra from NP-paper in Supplementary Fig. S3a). As shown in the optical micrographs of Figure $3 \mathrm{a}$, the extinction values are linear with the concentration of the CV solution from $10 \mu \mathrm{M}$ to $500 \mu \mathrm{M}$, but they are indistinguishable at concentrations less than $10 \mu \mathrm{M}$ due to the relatively high extinction of the silver nanoislands. By contrast, the $\lambda_{\text {LSPR }}$ becomes linearly red-shifted from $500 \mathrm{~nm}$ to $510 \mathrm{~nm}$ in wavelength (inset of Figure 3a) as the CV concentration increases from $50 \mathrm{nM}$ to $10 \mu \mathrm{M}$. The $\Delta \lambda_{\text {LSPR }}$ measurement enables an LOD of $50 \mathrm{nM}$, corresponding to $2 \mathrm{pg}$ per unit spot (see experimental details in the section on Materials and methods). In particular, the LOD of $\Delta \lambda_{\text {LSPR }}$ is also comparable to that of SERS by using NP-paper with $1.91 \times 10^{7}$ in the SERS EF (Supplementary Fig. S3b and also see the calculation procedures for SERS EF in the section on Materials and methods). Subsequently, NP-paper facilitates the scalable quantification and identification for small molecules in a broad range of molar concentrations, i.e., $50 \mathrm{nM}$ to $500 \mu \mathrm{M}$, by combining the LSPR wavelength shift and the extinction measurements. NP-paper also exhibits substantial MEF of small molecules. Figure 3b (top panel) displays the fluorescent images of four different dye molecules (TB, CR, SO, and FITC) at $500 \mathrm{nM}$ depending on the emission wavelength. The experimental results show that the NP-paper changes the average fluorescence intensity by factors of $0.9,3,6$, and 8 for 500 nM FITC, SO, $\mathrm{CR}$, and $\mathrm{TB}$, respectively, relative to the values achieved using normal chromatography paper (see experimental details in the section on Materials and methods). The difference in the fluorescence enhancement of dye molecules mainly results from the spectral reflectance $\left(R_{\mathrm{NP}-\text { paper }} / R_{\text {paper }}\right)$ of the silver nanoislands on the NP-paper (Figure $3 \mathrm{~b}$ bottom). In other words, the scattering component of the extinction spectrum for the silver nanoislands on NP-paper substantially contributes to the MEF due to the coupled fluorescence emission through the silver nanoislands resulting from the energy transfer of the excited fluorophore to the localized surface plasmon, whereas the absorption portion near $494 \mathrm{~nm}$ of $\lambda_{\text {LSPR }}$ impedes the excitation of fluorescence. For instance, FITC on NP-paper shows a fluorescence intensity that is slightly lower than that of normal chromatography paper, although both CR and SO exhibit substantial increases due to their higher spectral reflectance. The experimental results demonstrate that the LOD is $5 \mathrm{nM}$ for CR and SO (Supplementary Fig. S4a) and even down to the attogram level (185 attograms; $5 \mathrm{pM}$ ) for TB, which is two orders of magnitude lower than that of normal paper (Supplementary Fig. S4b). The calculation procedures for the LOD are discussed in the section on Materials and methods.

The rapid screening of mixed small molecules has been achieved by combining nanoplasmonic sensing with chromatographic separation. Figure $4 \mathrm{a}$ demonstrates a clear partitioning of concentrated dye molecules ( $50 \mathrm{mM} \mathrm{CR}, 40 \mathrm{mM} \mathrm{TB}$, and $5 \mathrm{mM} \mathrm{CV}$ ) in a mixture after NPpaper based ascending chromatography (see experimental details in the section on Materials and methods). The absorbance spectra of the analyte molecules were then measured at different positions (chromatograms) over NP-paper. The absorption bands of CR, TB, and CV were clearly distinguished at positions 1,2 , and 3 , respectively. In addition, the SERS spectra of the individual analytes also confirm the molecular 
fingerprints at each chromatogram. Note that a highly concentrated solution leads to the band broadening of each chromatogram due to the large diffusion of small molecules from the solvent to the NP-paper. Figure $4 \mathrm{~b}$ also demonstrates the ultrasensitive detection of separated small molecules at the attogram level (185 ag for TB). In this experiment, mixed small molecules at low concentrations of $15 \mathrm{nM} \mathrm{CR}, 15 \mathrm{pM}$ $\mathrm{TB}$, and $15 \mathrm{nM}$ SO were separated within $15 \mathrm{~min}$ and clearly identified at their fluorescence emission bands (also see Supplementary Fig. S6 and experimental details in the section on Materials and methods). The retention factors of the individual solutes were $0.13,0.48$, and 0.96 for $\mathrm{CR}, \mathrm{TB}$, and SO, respectively. Finally, the SERS assay of three different antioxidant vitamins was successfully performed after the chromatographic separation for $9 \mathrm{~min}$. Figure $4 \mathrm{c}$ shows the SERS intensity map of a single water-soluble vitamin (riboflavin) and two fat-soluble vitamins ( $\beta$-carotene and $\alpha$-tocopherol), depending on the migration distance over NP-paper. The retention factors were $0.25,0.92$, and 0.96 for riboflavin, $\beta$-carotene, and $\alpha$-tocopherol, respectively. In particular, riboflavin exhibited strong autofluorescence at $530 \sim 600 \mathrm{~nm}$ (inset of Figure 4c). The SERS LODs were $10 \mathrm{ng}$ for riboflavin, $537 \mathrm{pg}$ for $\beta$-carotene, and $11 \mathrm{ng}$ for $\alpha$-tocopherol. Both the rapid separation and the low-power SERS measurements successfully allow reliable label-free assays for mixed antioxidant vitamins (see experimental details in the section on Materials and methods).

\section{CONCLUSIONS}

To conclude, NP-paper offers a new optical readout platform for small molecules in a mixture using colorimetric, fluorescence, and SERS signals after the chromatographic separation of mixed small molecules. NP-paper provides many advantages over conventional paper chromatography because it enables the direct analysis of mixture samples and the label-free detection of diverse small molecules with ultrahigh sensitivity and because it has a rapid analysis time (separation plus detection) of $<15$ min with a small sample volume of $0.1 \mu \mathrm{L}$ and an ultra-low cost. The precise implementation of highly dense plasmonic nanostructures at the wafer level enables the specific identification of small molecules with extreme sensitivity and opens a new avenue for quantitative paper assays. This NP-paper assay can provide a huge potential for the rapid separation and label-free detection of diverse biomolecules in body fluids such as blood serum, cerebrospinal fluid, urine, and tear fluid. Paper nanoplasmonics can also be extended for high-throughput multiplex assays or functional assays in diverse research areas such as pathology, forensic science, and pharmacology.

\section{ACKNOWLEDGEMENTS}

This work is supported by the National Research Foundation of Korea (NRF) grant funded by the Korea government (MEST) (2014022751, 2014039957, 2011-0031866). This research was supported by a grant of the Korea Health Technology R\&D Project through the Korea Health Industry Development Institute (KHIDI), funded by the Ministry of Health \& Welfare, Republic of Korea (grant number: HI13C2181).

1 Gottesfeld JM, Neely L, Trauger JW, Baird EE, Dervan PB. Regulation of gene expression by small molecules. Nature 1997; 387: 202-205.

2 Stockwell BR. Exploring biology with small organic molecules. Nature 2004; 432 : 846-854.

3 Schreiber SL. Small molecules: the missing link in the central dogma. Nat Chem Biol 2005; 1: 64-66.

4 Ames BN, Shigenaga MK, Hagen TM. Oxidants, antioxidants, and the degenerative diseases of aging. Proc Natl Acad Sci USA 1993; 90: 7915-7922.
5 Jackson TM, Ekins RP. Theoretical limitations on immunoassay sensitivity: current practice and potential advantages of fluorescent $\mathrm{Eu}^{3+}$ chelates as non-radioisotopic tracers. J Immunol Methods 1986; 87: 13-20.

6 Kaddurah-Daouk R, Kristal BS, Weinshilboum RM. Metabolomics: a global biochemical approach to drug response and disease. Annu Rev Pharmacol Toxicol 2008; 48: 653-683.

7 Espada A, Molina-Martin M. Capillary electrophoresis and small molecule drug discovery: a perfect match? Drug Discov Today 2012; 17: 396-404.

8 Dentinger BTM, Margaritescu S, Moncalvo J-M. Rapid and reliable high-throughput methods of DNA extraction for use in barcoding and molecular systematics of mushrooms. Mol Ecol Resour 2010; 10: 628-633.

9 Consden R, Gordon AH, Martin AJ. Qualitative analysis of proteins: a partition chromatographic method using paper. Biochem J 1944; 38: 224-232.

10 Fletcher J. Filter-paper dot-immunobinding assay for detection of spiroplasma-citri. App/ Environ Microbiol 1987; 53: 183-184.

11 Heberling RL, Kalter SS. Rapid dot-immunobinding assay on nitrocellulose for viral antibodies. J Clin Microbiol 1986; 23: 109-113.

12 Martinez AW, Phillips ST, Butte MJ, Whitesides GM. Patterned paper as a platform for inexpensive, low-volume, portable bioassays. Angew Chem Int Ed 2007; 46: $1318-1320$.

13 Hossain SMZ, Luckham RE, Smith AM, Lebert JM, Davies LM et al. Development of a bioactive paper sensor for detection of neurotoxins using piezoelectric inkjet printing of sol-gel-derived bioinks. Anal Chem 2009; 81: 5474-5483.

14 Ali MM, Aguirre SD, Xu Y, Filipe CDM, Pelton R et al. Detection of DNA using bioactive paper strips. Chem Commun 2009; 6640-6642.

15 Chen H, Kou X, Yang Z, Ni W, Wang J. Shape- and size-dependent refractive index sensitivity of gold nanoparticles. Langmuir 2008; 24: 5233-5237.

16 Lei DY, Li J, Fernández-Domínguez AI, Ong HC, Maier SA. Geometry dependence of surface plasmon polariton lifetimes in nanohole arrays. ACS Nano2009; 4: 432-438.

17 Jensen TR, Duval ML, Kelly KL, Lazarides AA, Schatz GC et al. Nanosphere lithography: effect of the external dielectric medium on the surface plasmon resonance spectrum of a periodic array of silver nanoparticles. J Phys Chem B 1999; 103: 9846-9853.

18 Zhang J, Fu Y, Liang D, Nowaczyk K, Zhao RY et al. Single-cell fluorescence imaging using metal plasmon-coupled probe 2 : single-molecule counting on lifetime image. Nano Lett 2008; 8: 1179-1186.

19 Park S-G, Ahn M-S, Oh Y-J, Kang M, Jeong Y et al. Nanoplasmonic biopatch for in vivo surface enhanced Raman spectroscopy. Biochip J 2014; 8: 289-294.

20 Duhr S, Braun D. Why molecules move along a temperature gradient. Proc Natl Acad Sci USA 2006; 103: 19678-19682.

21 Kang T, Hong S, Choi Y, Lee LP. The effect of thermal gradients in SERS spectroscopy. Small 2010; 6: 2649-2652.

22 Kasera S, Biedermann F, Baumberg JJ, Scherman OA, Mahajan S. Quantitative SERS using the sequestration of small molecules inside precise plasmonic nanoconstructs. Nano Lett 2012; 12: 5924-5928.

23 Oh Y-J, Park S-G, Kang M-H, Choi J-H, Nam Y et al. Beyond the SERS: Raman enhancement of small molecules using nanofluidic channels with localized surface plasmon resonance. Small 2011; 7: 184-188.

24 Park M, Oh Y-J, Park S-G, Yang S-B, Jeong K-H. Electrokinetic preconcentration of small molecules within volumetric electromagnetic hotspots in surface enhanced Raman scattering. Small 2015; 11: 2487-2492.

25 Lee CH, Hankus ME, Tian L, Pellegrino PM, Singamaneni S. Highly sensitive surface enhanced Raman scattering substrates based on filter paper loaded with plasmonic nanostructures. Anal Chem 2011; 83: 8953-8958.

26 Yu WW, White IM. Chromatographic separation and detection of target analytes from complex samples using inkjet printed SERS substrates. Analyst 2013; 138: 3679 3686.

27 Tseng S-C, Yu C-C, Wan D, Chen H-L, Wang LA et al. Eco-friendly plasmonic sensors: using the photothermal effect to prepare metal nanoparticle-containing test papers for highly sensitive colorimetric detection. Anal Chem 2012; 84: 5140-5145.

28 Polavarapu L, Porta AL, Novikov SM, Coronado-Puchau M, Liz-Marzán LM. Pen-onpaper approach toward the design of universal surface enhanced Raman scattering substrates. Small 2014; 10: 3065-3071.

29 Campbell CT. Ultrathin metal films and particles on oxide surfaces: structural, electronic and chemisorptive properties. Surf Sci Rep 1997; 27: 1-111.

30 Le Ru EC, Blackie E, Meyer M, Etchegoin PG. Surface enhanced Raman scattering enhancement factors: a comprehensive study. J Phys Chem C 2007; 111: 1379413803.

31 Kang M, Kim J-J, Oh Y-J, Park S-G, Jeong K-H. A deformable nanoplasmonic membrane reveals universal correlations between plasmon resonance and surface enhanced Raman scattering. Adv Mater 2014; 26: 4510-4514.

This work is licensed under a Creative Commons Attribution-

NonCommercial-NoDerivs 4.0 Unported License. The images or other third party material in this article are included in the article's Creative Commons license, unless indicated otherwise in the credit line; if the material is not included under the Creative Commons license, users will need to obtain permission from the license holder to reproduce the material. To view a copy of this license, visit http://creativecommons.org/licenses/ by-nc-nd/4.0/ 